\title{
Using master tickets as a storage for problem-solving expertise
}

\author{
Gabi Dreo \\ University of Munich, Department of Computer Science \\ Leopoldstr. 11B, 80802 Munich, Germany \\ email:dreo@informatik.uni-muenchen.de \\ Robert Valta \\ Leibniz-Rechenzentrum \\ Barerstr. 21, 80333 Munich, Germany \\ email:valta@lrz-muenchen.de
}

\begin{abstract}
Heterogeneity and distribution of communications services and resources impose new requirements on fault management. Support staff performing fault diagnosis has to be supported with sophisticated tools, like enabling a simple and fast access to problem-solving expertise. This paper presents an approach for the storage and retrieval of problem-solving expertise by introducing the concept of a master ticket. The idea is to generalize information about a fault and store this information in a master ticket. Problem-solving expertise is obtained by the retrieval and the instantiation of a useful master ticket. A structure on the master ticket repository is defined by specifying relationships between master tickets, which guide the operator throughout fault diagnosis and fault recovery. The usability of the proposed concept is verified using a prototype.
\end{abstract}

\section{Keywords}

Distributed Systems, Fault Diagnosis, Trouble Ticket Systems

\section{Introduction}

As the heterogeneity, complexity, and distribution of communications resources, services, and applications continue to grow, the importance of being able to manage such complex environments increases correspondingly (e.g., [HeAb 94]). To cope with these requirements, new sophisticated functionalities and advanced tools to provision, manage, and maintain the network are needed. This becomes especially obvious in the area of fault management, which generally comprises fault detection, fault diagnosis, and fault recovery. 
Fault management in such a heterogeneous environment has to deal with the specialization of the personnel maintaining the network, the great amount of alarms issued from a network management platform, and the ambiguous, incomplete information reported from end users in case of recognizing a trouble. Resulting potential problems are (i) difficult access to problemsolving expertise, mostly hidden in the "heads" of a few experts, (ii) the flooding of experts with events from a network management platform, and (iii) the ambiguity and incompleteness of information reported from end users.

Trouble Ticket Systems (TTSs) have been introduced to assist during all phases of fault management. Information entered and activities performed during the fault management process are documented in a trouble ticket. Basic functions of a TTS include the means for trouble ticket management and the coordination of maintenance, repair, and testing activities (e.g., [RFC 1297]). Beside the basic functions of trouble management, as described in (e.g., [ITU-T 92], [ANSI 92], [NMF 92b]), the necessity for more sophisticated functions has been recognized. For example, in [NMF 92a] the need for building knowledge databases from user experience, in [LeDr 93] the extension of TTSs to fault diagnosis, and in [VaJa 93] the deployment of group communication techniques in network management were discussed.

This paper tackles the problem of improving the general access to problem-solving expertise by introducing the concept of a master ticket. The idea of the master ticket concept is to generalize information about a fault and store this information in a master ticket. Problemsolving expertise for an outstanding trouble ticket is obtained by the retrieval and the instantiation of a useful master ticket. The concept of a master ticket and the relationships defined between master tickets provide a kind of a "structure" on a trouble ticket repository.

Problem-solving is a vital research topic in artificial intelligence (e.g., [Hinr 92], [Stee 90], [Aamo 91], [Koto 89]). Recently, the applicability of case-based reasoning to fault management has been investigated, for example in [Lewi 93]. The key point of this approach is to retrieve problem-solving expertise by searching for a trouble ticket which is "similar" to an outstanding ticket. The diagnostic and repair activities performed for this ticket are applied to the outstanding ticket. Difficulties of this approach are the definition of the determinators that record relevance information, and the similarity relations between trouble tickets.

The paper proceeds as follows: First, the concept and the structure of a master ticket are outlined. Subsequently, the generation and application of master tickets for the storage and retrieval of problem-solving expertise are presented. Relationships between master tickets are pointed out. In addition, we discuss the usability of the master ticket approach for the correlation of trouble tickets. A description of the prototype follows. Finally, some concluding remarks and further work are stated.

\section{Master Ticket Concept}

\subsection{Motivation and requirements}

Due to the heterogeneity of services and resources, the specialization of personnel becomes an evident problem. The motivation for the introduction of the master ticket concept results from the requirement to enable fast and simple access to problem-solving expertise. Beside this, organizational support of fault diagnosis has to be provided. When developing a concept for the access to problem-solving expertise, the integration with the network management environment has to be considered as well. 
Requirements for a concept of problem-solving expertise can be structured with respect to the acquisition, storage, and retrieval of problem-solving expertise, and are as follows:

- The acquisiton of problem-solving expertise should be simple and proceed as much as possible automatically from the documented fault information in trouble tickets (cases).

- Problem-solving expertise should be stored in a structured library. The structure should be realized through generalized fault information. Fault-specific information is in the generalized form represented with parameters.

- The number of retrieval steps to obtain useful problem-solving expertise should be minimal.

\subsection{The idea}

To meet the stated requirements, we propose the concept of a master ticket. The idea of the master ticket is to structure the trouble ticket repository under the viewpoint of generalizing information about a fault (Fig. 1). Information, like symptoms, diagnostic activities, and repair activities is stored in a master ticket in a generalized form. Generalization means that failurespecific information, like user information, addresses of nodes or topology data contained in trouble tickets is replaced with parameters in a master ticket. An example of the information held in a master ticket would be is_active(\$process, \$node), where \$process represents a process, \$node the hostname or IP address of a computer system, and is_active() a diagnostic activity which tests whether the specified process is running on the host.

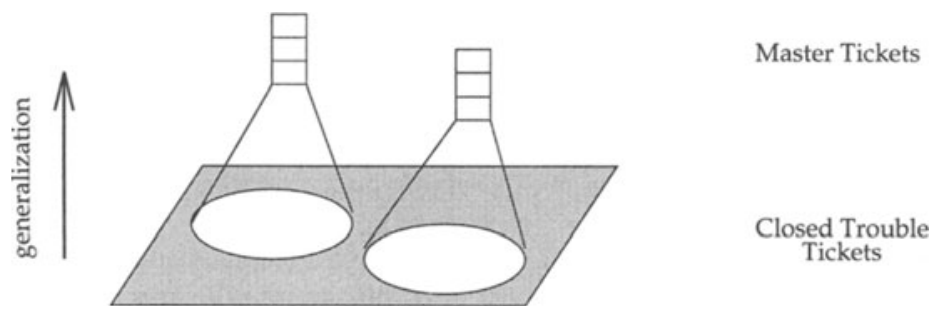

Figure 1: Master ticket concept

Retrieving problem-solving expertise is the search for an adequate master ticket. The retrieval proceeds in two steps. First, an adequate master ticket has to be determined, and second, this master ticket has to be instantiated. To instantiate a master ticket means to substitute, for example, the parameter \$node in the previous example with an IP address and the parameter \$process with the name of a process. Thus, the result would be to apply is_active( "named", "129.187.10.32") as a diagnostic activity for an outstanding trouble ticket.

During fault recovery, the state of a trouble ticket switches from open, including only the symptom, to closed, including also the diagnostic activities taken, the identified fault, and the repair activities performed. If the search for a useful master ticket fails (i.e., the fault type has not yet appeared), the open trouble ticket has to be solved solely by an expert. Afterwards, the 
master ticket repository is updated with a new master ticket for this fault. The update of the master ticket repository proceeds also if new activities for existing faults are encountered.

To summarize, the master ticket concept consists of two steps:

1. the generation of master tickets, and the

2. application of master tickets. Subsidiary steps of the application are:

- the retrieval of a useful master ticket, and the

- instantiation of this master ticket.

\subsection{Structure of the master ticket}

Recalling that a master ticket contains generalized information about a fault, the information contained in a master ticket is as follows:

Master_ticket $=[\operatorname{symptom}(p)$, diagnostic_activity $(p)$, fault $(p)$, repair_activity $(p)]$,

where $p$ is an abbreviation for parameters. The first item in the master ticket is a symptom (i.e., trouble report). When considering trouble reports which are issued by end users, the symptom includes the description of the service used and whether the service (i) was not provided or (ii) not provided with the requested Quality of Service (QoS). The idea behind this classification is to decompose the symptom information into elements that allow the retrieval of a master ticket and the instantiation of a master ticket. For the retrieval of a master ticket, the service used and the classification is sufficient. However, information such as the end user who has reported the trouble and the time the trouble was recognized is of importance for the instantiation.

The parameters in the master ticket have to be substituted with concrete values. Substitution of parameters can be done in several ways:

- The operator who is diagnosing the fault retrieves the values for the parameters from the problem description provided by the end user who reported the problem.

- The operator contacts the end user to get information which cannot be retrieved from the problem description.

- The operator retrieves data from management databases, for example from an inventory system, to map a user account to the name of a user or a user location to the name of a printer.

- The operator might access the client node to retrieve client specific configuration parameters, for example the default printer.

The second item in the master ticket describes the diagnostic activity taken to diagnose the fault, which is described in the third item of the master ticket. The fourth item describes the repair activity which should be performed to recover from the diagnosed fault.

Examples of master tickets are as follows:

Master_ticket $_{i}=[$

no_printing_output

( $\$$ client $=<$ name of node where user starts the print job $>$, 


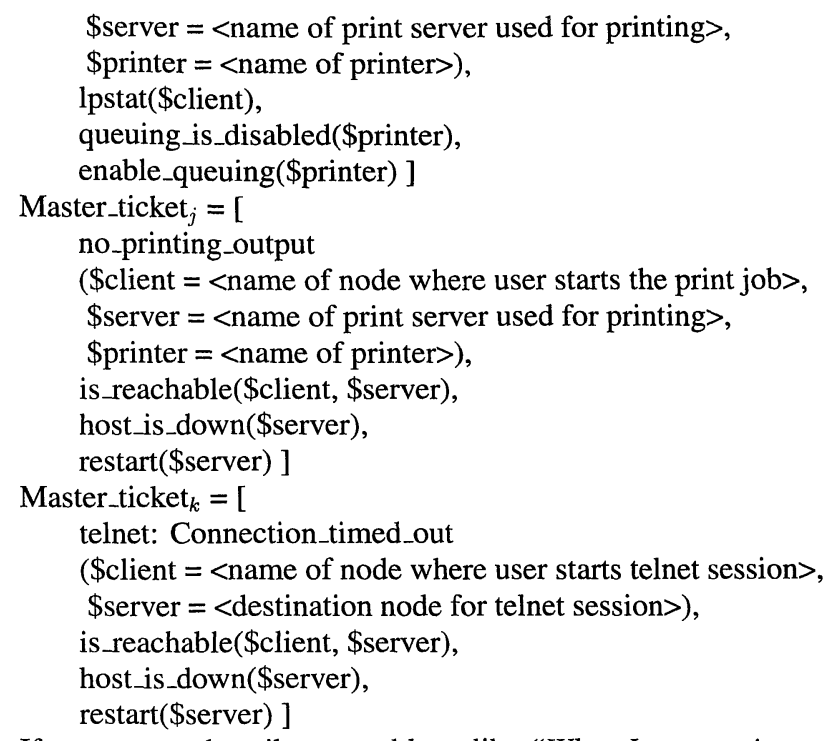

If now a user describes a problem, like "When I try to print a report from my workstation sun12 at our department printer, there is no output", such a problem would first lead to the retrieval of Master_ticket ${ }_{i}$. After its instantiation, the diagnostic activity lpstat(sun12) is performed. If this activity reveals no problem, another master ticket - Master_ticket ${ }_{j}$ - is retrieved. The diagnostic activity is_reachable(sun12, sun-department) is performed, which shows that node sun-department crashed. Thus, the problem can be solved by restarting node sun-department.

An important design issue regarding the contents of a master ticket is the level of specificity used to describe the diagnostic and repair activities. In the above example, a diagnostic activity is stated as is_reachable (\$client, \$server). Such a statement leaves some freedom about how reachability between a client node and a server node is really tested (e.g., using a ping or a traceroute command or checking the status information provided by a management station). However, an activity could be specified more precisely if required, which would decrease the presumed level of expertise for staff members.

\subsection{Relationships between master tickets}

In today's distributed environments many end user services rely on a hierarchy of underlying services. A distributed application (e.g., remote printing) depends on client, server and gateway processes, which themselves depend on system software and hardware. For communication between processes a transport network is required. Transit networks, networking devices and communication links must be properly configured and in operating state. Furthermore, many services rely on other distributed services, e.g., name resolution provided by a distributed name server. This hierarchical structure affects fault diagnosis because the underlying service hierarchy can be tested in a top-down or bottom-up strategy to isolate a fault whenever a problem is reported. 
For our master ticket approach this has several consequences. We have to avoid a complete, exhaustive diagnosis of a service-related problem within a single master ticket for that service, because that would lead to a high redundancy (i.e., testing the transport network would be represented in all master tickets for distributed services). Instead, we not only provide master tickets for user services but also for the underlying services within our service hierarchy. As easily recognized, the service hierarchy implies a corresponding hierarchy between master tickets for the different services. For example, if a service $A$ relies on a service $B$, applying master ticket $A$ might lead us to the conclusion that the problem might be caused by service $B$. Thus, we can start to work on that problem by using the master ticket for service $B$.

This raises the question of how relationships between services - and thereby relationships between master tickets - should be handled within our master ticket approach:

1. Based on a framework for distributed applications we can model a service hierarchy and derive a corresponding model for our master tickets. An example of such a framework is presented in [HNG 94], which consists of application services, application-oriented services, basic distributed services, and communications services.

2. We can define relationships between master tickets in a more pragmatic way according to the procedures followed during fault diagnosis.

We decided to choose the second approach because experience shows us that it is rather difficult to define a common service architecture for an existing heterogeneous environment.

In general, the process of fault diagnosis is iterative. The availability or quality of a service is tested by testing the availability or quality of the underlying services. Testing itself is in many cases nothing else but trying to use an underlying service. In such a case the tester behaves like a normal user of the underlying service. Master tickets are therefore related by interpreting diagnostic activities as usage of a service. Relationships between master tickets are defined as follows:

- A diagnostic activity within a master ticket is interpreted as usage of a service (i.e., ping as a diagnostic activity is interpreted as usage of an IP reachability service).

- Failure of a diagnostic activity leads to a new trouble ticket, called Internal Trouble Ticket (ITT), which can be further diagnosed by searching for a new master ticket.

To make sure that the diagnosis process terminates, we distinguish between

1. Core master tickets, which contain a fault and a repair activity.

If the diagnostic activity of a core master ticket fails, we immediately know the fault and how to repair it (e.g., if the diode labeled cpu on a router's front panel is red, the cpu board is malfunctioning and has to be replaced).

2. Relational master tickets, which do not contain a fault and a repair activity.

If the diagnostic activity of a relational master ticket fails (e.g., brouter bro4cz could not be reached), we have not yet identified the fault. We have to continue with the diagnosis process by creating a new internal trouble ticket which is further diagnosed by retrieving a new master ticket. Thus, relational master tickets are only "pointers" leading to other relational master tickets or finally to a core master ticket (Fig. 2). 


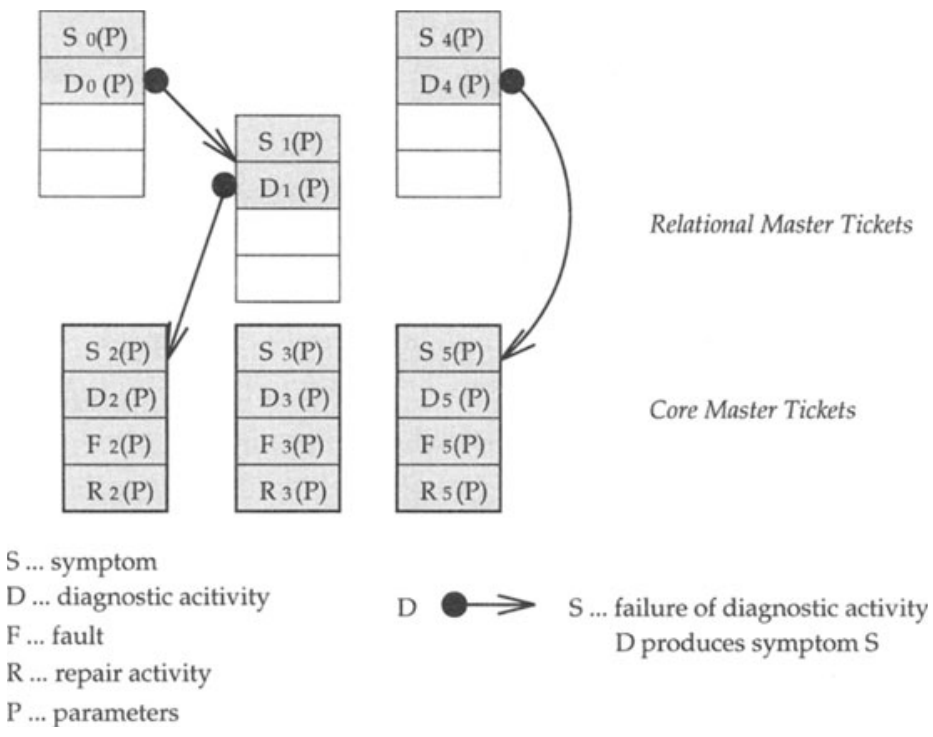

Figure 2: Relationships between master tickets

\section{Generation of Master Tickets}

\subsection{Requirements on the trouble ticket structure}

The structure of a master ticket depends to a great extent on the structure of a trouble ticket. The term "structure" of a trouble ticket means the set of fields in the trouble ticket schema, and the set of predefined selection values for each field. A trouble ticket is completely structured if information about a trouble is entered completely via predefined selection values. Requirements on the structure of a trouble ticket are stated from

1. users or help-desk staff who prefer free-form text when describing a problem and how it was solved, and

2. the procedure for the creation of new master tickets, which requires formalized and structured trouble tickets.

These requirements are almost opposite to each other. Thus, an extensive analysis of the trouble ticket structure, still acceptable by the users of a TTS, but supporting also the master ticket concept is of great importance.

Our experiences, gained in one year of usage of TTSs at the computing center, have shown that the acceptance of a TTS by the users depends to a great extent on the efficiency and speed of entering information about a problem. A desire is that the information entered should be precise, complete, and as unambiguous as possible. Unfortunately, personnel documenting the reported 
problems just want to enter the information as it is reported, and do not want to structure it. There are various reasons for this, like lack of time, knowledge or experience.

Realizing these problems we have provided support to the personnel by enabling a lot of information to be entered automatically by the system. For example, an assignee for an open trouble ticket is determined automatically according to the service specified and availability. We are developing a hypertext based tool, called "Intelligent Assistant", which provides very flexible and fast access to various databases, and guides the operator during the entering of information.

To fill the gap between the structure of a trouble ticket as required by the support staff and as needed by the master ticket concept, a formalization of a trouble ticket is necessary. The formalization function transforms a user trouble ticket, containing free-form descriptions, to a formalized user trouble ticket used further in the master ticket concept. Parsing the free-form description of the symptom should be performed with sophisticated lexical text analysis. If not stated explicitly otherwise, we are considering only formalized trouble tickets for the remainder of the paper.

The structure of a formalized user trouble ticket as required by the master ticket concept is shown in Fig. 3.

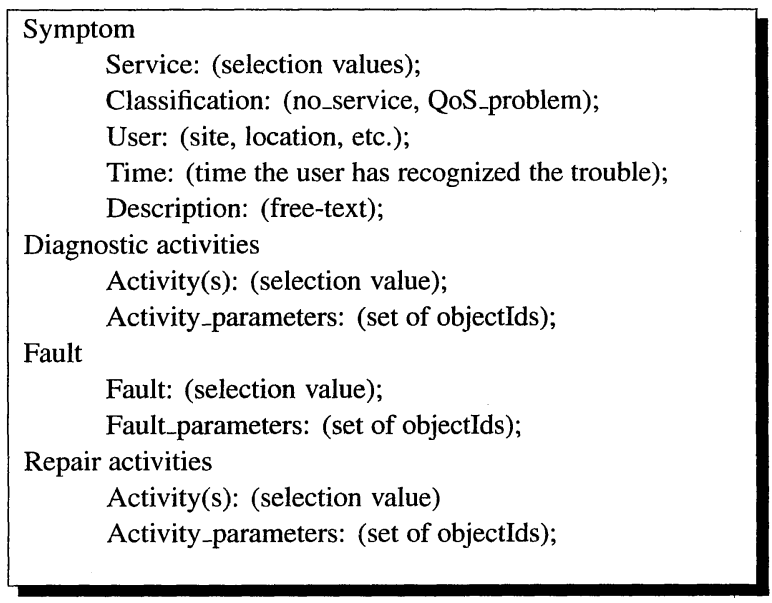

Figure 3: Structure of a formalized trouble ticket

\subsection{Generation procedure}

The generation of master tickets proceeds in two steps:

- generation of core master tickets based on product descriptions (e.g., in case a new device or application is incorporated in the network), and

- generation of core or relational master tickets based on closed trouble tickets. 
The first step is performed by experts analyzing the documentation of the products and identifying the documented faults, diagnostic and repair activities.

If during the retrieval of a master ticket no useful master ticket could be obtained, an expert has to proceed with fault diagnosis without access to problem-solving expertise. During fault recovery he documents all performed diagnostic activities in the current trouble ticket. After fault recovery, the update procedure is started to generate master tickets (relational and core) for this closed trouble ticket. The updated procedure is as follows:

1. First, it is checked if a core master ticket exists for the fault diagnosed in the closed trouble ticket. If this is true, new diagnostic activities must be added to the master ticket repository by defining new relational master tickets. Note, this situation occurs if a new symptom or diagnostic activity is identified for an already documented fault.

2. In case a core master ticket could not be identified for the diagnosed fault, a new core master ticket has to be generated. Part of the information contained in the closed trouble ticket (e.g., the diagnostic activities identifying the fault, the fault itself, and the repair activities) is included in the core master ticket. The symptom, and the diagnostic activities leading to the core master ticket are included in the relational master tickets. During the generation of the relational master tickets, it is checked whether some of them already exist.

Concrete values, like IP addresses of nodes, in the closed trouble ticket are replaced with parameters in the master tickets.

\section{Application of the Master Ticket Approach}

For the application of the master ticket approach the key points are the efficiency of the usage and the acceptance of the concept. Accessing problem-solving expertise in the proposed concept means (i) to retrieve a useful master ticket, and (ii) to instantiate this master ticket.

The retrieval of a useful master ticket can be performed with more or less sophisticated methods. The easiest way is simple pattern matching between the symptom contained in the open trouble ticket and the symptoms contained in the master tickets. Thus, for a given trouble ticket $\left(T T_{1}\right)$ with a symptom $S_{1}(V)$, master tickets $M T_{1}, \ldots, M T_{n}$ with the same symptom information are retrieved. Then, for each master ticket $M T_{i}, \mathrm{i}=1 \ldots \mathrm{n}$, the following steps are performed (Fig. 4):

1. All parameters of the master ticket are substituted.

2. The diagnostic activity $D_{i}$ of master ticket $M T_{i}$ is executed with all parameters replaced by the previously determined values.

3. If the diagnostic activity does not fail, i.e., it gives us no indication of the cause of the problem, the next master ticket is worked on.

4. If the diagnostic activity fails, we have to check whether a fault is defined for this diagnostic activity: 


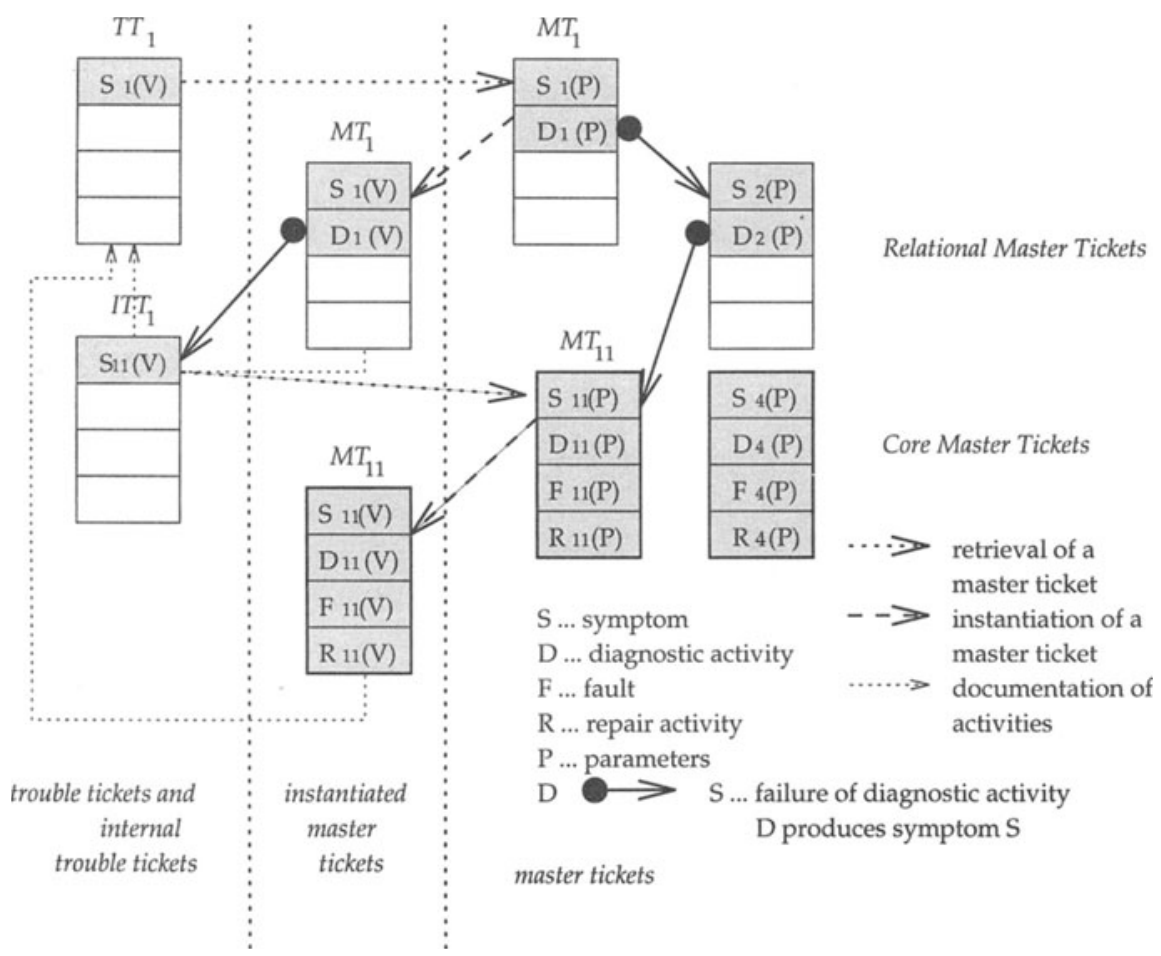

Figure 4: Application of the master ticket concept

(a) If there is no fault associated with the diagnostic activity, a new internal ticket $I T T_{1}$ which describes the negative test result as a failure of the usage of the underlying service is created.

The new internal ticket $I T T_{1}$ is then diagnosed by searching for a corresponding master ticket (e.g., $M T_{11}$ ) for the indicated service failure.

(b) If there is a fault (and a repair activity), we instantiate the fault and the repair activity. The repair activity is presented to the support staff and can be executed. The algorithm terminates.

5. If no master ticket could be retrieved, no problem-solving expertise is available for the symptom. An expert has to proceed with fault diagnosis on his own. He documents all performed diagnostic and repair activities and the identified fault in the current trouble ticket. This information is later used to create a new master ticket for the currently unknown symptom. Furthermore, the range of available diagnostic activities should be offered to him as a help. 


\section{Correlation in the Master Ticket Approach}

In addition to recording problem-solving expertise, the master ticket approach provides a mechanism for correlating trouble tickets. Correlation is defined as the grouping of trouble tickets that are associated with the same fault. A benefit of correlation is that it prevents multiple diagnoses of the same fault.

During the application of the master ticket graph the fault diagnosis process can produce a sequence of internal trouble tickets (Fig. 4), like

$$
T T_{1} \rightarrow I T T_{11} \rightarrow I T T_{12} \rightarrow I T T_{13}
$$

where $T T_{1}$ means an open trouble ticket, and $I T T_{1 i} \mathrm{i}=1, \ldots, 3$ are the internal tickets obtained during retrieval and instantiation. Assuming another user has reported a trouble some minutes later than the first one, then the associated sequence of internal trouble tickets would be as follows:

$$
T T_{2} \rightarrow I T T_{21} \rightarrow I T T_{22} \rightarrow I T T_{23}
$$

The sequence of internal trouble tickets provides traces of the fault localization process. If during fault diagnosis common internal trouble tickets can be identified (e.g., $I T T_{12}=I T T_{23}$ ), then the originating trouble tickets $T T_{1}$ and $T T_{2}$ can be considered to be correlated. The comparison of sequences of internal trouble tickets is performed solely on a syntactical basis.

If such common internal trouble tickets could be identified, it can be decided to continue work only on one sequence of internal trouble tickets. The most promising way is to continue work with the sequence including information which have been reported from a person with high domain knowledge.

The proposed approach provides a simple but efficient method to correlate new incoming trouble reports with existing tickets. The existing tickets may or may not be already in the process of fault diagnosis.

\section{Design of MASTER}

The master ticket concept is currently implemented in a prototype, called MASTER, on the Application Programming Interface of the Action Request System from Remedy (version 1.2). The ARS is used by the hot line of the computing center and for research purposes at the university. The runtime environment of MASTER is shown in Fig. 5.

The core of MASTER are the programs for the text analysis, generation, instantiation, and retrieval of master tickets using the ARS API.

We use the following schemas: the trouble ticket schema, the formalized trouble ticket schema, the internal trouble ticket schema, and the master ticket schema. The trouble ticket schema is used by the hot line of the computing center to document trouble reports. The implementation of the formalization function is currently based on lists of negative and positive keywords. The formalized trouble ticket schema is presented to an operator as a proposal who can check the validity of the formalization. A more sophisticated text analysis could minimize the interventions of the operator. The retrieval and the instantiation of master tickets are implemented with the available ARS mechanisms, like active links or macros, and programs using the ARS API. 


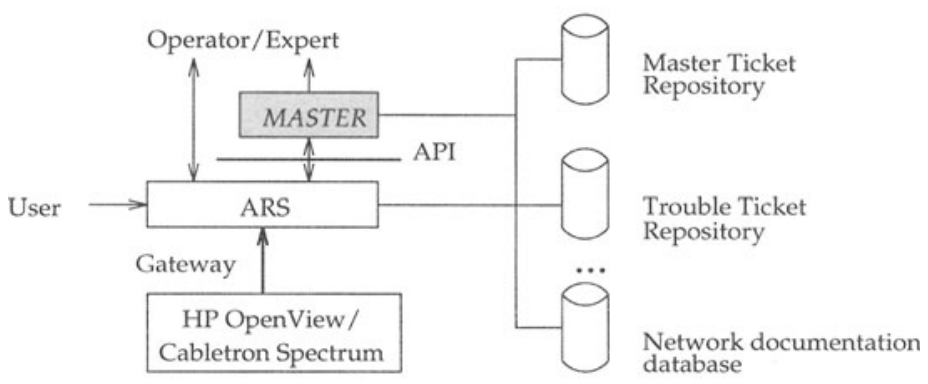

Figure 5: Environment of MASTER

First experimental results with the prototype have shown promising results. Of course, an extensive usage of the prototype at the computing center will answer the question whether the system will render fault management more efficient and less time-consuming.

\section{Conclusions and Further Work}

Heterogeneity and distribution of communications services and resources impose new requirements on fault management. Due to the specialization of the personnel maintaining the network, the access to problem-solving expertise is a vital research topic in fault management. In this paper, a solution for this problem is presented by introducing the concept of a master ticket. The idea of the master ticket approach is to generalize information about a fault and store it in a master ticket. Problem-solving expertise is obtained by the (i) retrieval of a useful master ticket, which is based on the procedure followed during fault diagnosis, and (ii) the instantiation of the useful master ticket.

Our further work will concentrate on the (i) development of various tools, like a tool for supporting the generation of master tickets, (ii) the feasibility of using a common service hierarchy to implement the relations between master tickets, and (iii) testing the prototype extensively at the computing center.

\section{Acknowledgements}

The authors wish to thank the members of the Munich Network Management (MNM) Team for helpful discussions and valuable comments on previous versions of the paper. The MNM Team is a group of researchers of the Munich Universities and the Bavarian Academy of Sciences. It is directed by Prof. Dr. Heinz-Gerd Hegering. We gratefully acknowledge in particular Bernhard Neumair, Victor Apostolescu, and Anja Schuhknecht, who provided valuable suggestions and advice. 


\section{References}

[Aamo 91] A. Aamodt, A knowledge-intensive approach to problem solving and sustained learning, Ph.D. dissertation, University of Trondheim, 1991.

[ANSI 92] ANSI, Operations, Administration, Maintanance, and Provisioning (OAM\&P)-Extension to Generic Network Model for Interfaces between Operations Systems across Jurisdictional Boundaries to support Fault Management - Trouble Administration, T1M1.5/92-01R2, 1992.

[HeAb 94] H.-G. Hegering and S. Abeck, Integrated Network Management and System Management, AddisonWesley, September 1994.

[Hinr 92] T.R. Hinrichs, Problem solving in open worlds, Lawrence Erlbaum Associates, 1992.

[HNG 94] H.-G. Hegering, B. Neumair and M. Gutschmidt, "Cooperative Computing and Integrated System Management - A Critical Comparison of Architectural Approaches", Journal of Network and Systems Management, 2(3), October 1994.

[INM-III 93] H.-G. Hegering and Y. Yemini, editors, Proceedings of the 3rd IFIP/IEEE IInternational Symposium on Integrated Network Management, San Francisco, IFIP, North-Holland, April 1993.

[ITU-T 92] ITU-T, Trouble Management Function - An overview, Question 24/VII, 1992.

[Koto 89] P. Koton, Using experience in learning and problem solving, Ph.D. dissertation, Massachusetts Institute of Technology, 1989.

[LeDr 93] L. Lewis and G. Dreo, "Extending Trouble Ticket Systems to Fault Diagnostics", IEEE Network Special Issue on Integrated Network Management, 7(6):44-51, November 1993.

[Lewi 93] L. Lewis, "A Case-Based Reasoning Approach to the Resolution of Faults in Communications Networks", In [INM-III 93], pages 671-682.

[NMF 92a] "ISO/CCITT and Internet Management: Coexistence and Interworking Strategy", Issue 1.0, Network Management Forum, October 1992.

[NMF 92b] "Application Services: Trouble Management Function", Issue 1.0, Network Management Forum, August 1992.

[RFC 1297] IAB, NOC Internal Integrated Trouble Ticket System, Functional Specification Wishlist, RFC 1297, January 1992.

[Stee 90] L. Steels, "Components of expertise", AI Magazine, 11(2):29-49, 1990.

[VaJa 93] R. Valta and R. de Jager, "Deploying Group Communication Techniques in Network Management", In [INM-III 93], pages 751-763.

\section{Biographies}

GABI DREO received B.S. and M.S. degrees in computer science from the University of Maribor, Slovenia. Currently, she is a Ph.D. student at the University of Munich and a member of the Munich Network Management team, directed by Prof. Dr. Heinz-Gerd Hegering, where she does research on integrated network and system management.

ROBERT VALTA received the degree of a Diplom-Informatiker in 1984 and the degree of a Dr.rer.nat. in 1990 both from the Technische Universität in Munich. He was a research staff member at the department of Computer Science of the Technische Universität and at the LeibnizRechenzentrum in Munich. In 1994 he joined Softlab GmbH where he is engaged in several network and system management projects. 